\title{
Results of a pilot antibiotic resistance survey of Albanian poultry farms
}

S.D. Alcaine ${ }^{a, *}$, L. Molla ${ }^{b}$, S.R. Nugen ${ }^{a}$, H. Kruse ${ }^{c}$

${ }^{a}$ Department of Food Science, University of Massachusetts, Amherst, MA, USA

${ }^{\mathrm{b}}$ Health \& Environment Department-Food Safety \& Nutrition Section, Institute of Public Health, Tirana, Albania

'Food Safety, Division of Communicable Diseases, Health Security, and the Environment, WHO Regional Office for Europe, Copenhagen, Denmark

\section{ARTICLE INFO}

Article history:

Received 23 July 2015

Accepted 10 November 2015

Keywords:

Antibiotic resistance

Poultry

Salmonella

Escherichia coli

Albania

* Corresponding author. Present address: Department of Food Science, 240 Chenoweth Laboratory, 100 Holdsworth Way, Amherst, MA 01003, USA. Tel.: +1 4147914952.

E-mail address: salcaine@umass.edu (S.D. Alcaine). 


\section{ABSTRACT}

Global dissemination of antibiotic-resistant bacteria in food animals is a major public health concern. Whilst many countries have implemented prudent antibiotic use policies and surveillance systems both in clinical and veterinary settings, there are no such systems in place in Albania and little is known about the levels of antibiotic-resistant bacteria in food animals within the country. A total of 172 poultry samples were taken from six Albanian farms over a 3-month period and were tested for the presence of Enterobacteriaceae. In total, 91 bacterial isolates were obtained and were characterised by species (Escherichia coli, Salmonella spp. or other Enterobacteriaceae) and by susceptibility to 11 antibiotics. Resistance rates of E. coli and Salmonella isolates were, respectively: amoxicillin (86\%, 64\%); chloramphenicol $(77 \%, 82 \%)$; ciprofloxacin $(93 \%, 73 \%)$; cefotaxime $(14 \%$, $0 \%)$; gentamicin (12\%, 0\%); kanamycin (30\%, $18 \%)$; nalidixic acid $(91 \%, 73 \%)$; streptomycin $(70 \%$, $55 \%)$; sulphonamides (91\%, 73\%); tetracycline (95\%, 73\%); and trimethoprim (79\%, 64\%). Multidrug resistance to at least four antibiotics was observed in $95 \%$ of E. coli isolates and $82 \%$ of Salmonella. In conclusion, these data indicate that: (i) Salmonella and E. coli isolates from Albanian poultry farms exhibit high to extremely high levels of antibiotic resistance; (ii) Salmonella and E. coli isolates exhibit resistance to multiple antibiotics; and (iii) multidrug resistance profiles among Enterobacteriaceae are geographically widespread. Implementation of prudent antibiotic use policies in food animals and related surveillance will be necessary to reduce the emergence, spread and establishment of highly resistant strains across poultry farms in Albania. 


\section{Introduction}

In response to rising rates of antibiotic resistance among clinical bacterial isolates and data linking antibiotic use to resistance development [1-3], leading public health organisations such as the World Health Organization (WHO), the US Centers for Disease Control and Prevention (CDC) and the Royal Society of Medicine have published recommendations calling for (i) prudent use of antimicrobials, (ii) improved surveillance of antimicrobial use and resistance and (iii) awareness campaigns both for health professionals and the public on the control and risks of antimicrobial resistance [4]. Research has also highlighted the public health impact of veterinary antibiotic use and the movement of antibiotic-resistant bacteria between animal and human populations $[5,6]$. Whilst antibiotics are commonly used to treat, prevent and control diseases in food animals as well as to promote growth, adequate data detailing the amount and frequency of antibiotic use in animal husbandry are lacking [7]. Institutions such as the European Food Safety Agency (EFSA), the European Centre for Disease Prevention and Control (ECDC), the European Surveillance of Veterinary Antimicrobial Consumption (ESVAC) and the US National Antimicrobial Resistance Monitoring System for Enteric Bacteria (NARMS) have been vital in illuminating the interplay between human and animal populations, antimicrobial use and antimicrobial resistance. The monitoring systems supported by these institutions, however, are primarily focused on Western Europe and North America, obscuring the global picture of antibiotic resistance. Research suggests that the prevalence of antibiotic-resistant bacteria is much higher in other regions [8]. Globalisation of the food supply chain and increased global travel means that antibiotic practices and the prevalence of antibioticresistant bacteria within these countries will have public health implications well beyond their borders. It is therefore critical to develop a more precise picture of global antibiotic resistance, to identify public health risks and to implement appropriate strategies for control where appropriate.

Albania is located in southeastern Europe and in 2014 it became an official candidate for accession to the European Union (EU). Albania currently lacks surveillance programmes for antibiotic consumption and resistance, thus it is difficult to gauge the potential health impacts of increasing travel and trade. Recent surveys have found poor control and misuse of clinically important antibiotics $[9,10]$. Agricultural exports are an important economic focus for Albania, but local food safety standards represent a hurdle for export growth [11]. There is little peer-reviewed published material on antibiotic use or levels of antibiotic-resistant bacteria in food animals in this country.

In this paper, we report the results of a short-term pilot surveillance study initiated as part of a joint WHO-World Organization for Animal Health (OIE) project on 'National Human and Animal Health Systems Assessment Tools and Bridges' to gain a preliminary understanding of bacterial resistance levels in food animals in Albania. This pilot survey looked at antibiotic resistance levels in Enterobacteriaceae, with a focus on Salmonella and Escherichia coli, isolated from six poultry farms over a 3-month period. To put these results in context, they were compared with the antimicrobial resistance levels in poultry reported by EFSA for the EU. We hope the results of this pilot survey will call attention to the antibiotic-related policies in Albania and support the long-term implementation of robust surveillance and prudent use programmes. 


\section{Materials and methods}

\subsection{Bacterial isolates}

All isolates included in this study were obtained as part of a field survey examining levels of antibiotic resistance in Albanian poultry farms. Isolates included in this study were cultured from 172 poultry samples of the neck skin, stomach, intestines, stomach and cloacal swabs from slaughtered poultry. Samples were collected from six Albanian farms during three visits to each farm from December 2013 to early February 2014 (Fig. 1). Samples were processed using ISO 6579: 2002 'Microbiology of food and animal feeding stuffs - horizontal method for the detection of Salmonella spp.' Subsequently, all colonies, including those that were not Salmonella-like on selective media, were characterised biochemically using API 20E (bioMérieux, Durham, NC). For the purposes of this study, isolates were classified as E. coli, Salmonella spp. or 'other Enterobacteriaceae' (e.g. Klebsiella spp., Citrobacter spp., etc.). All Salmonella spp. were serotyped. A total of 91 bacterial isolates were cultured from the 172 samples, including 3 samples that yielded 2 isolates each. There were 84 negative samples.

\subsection{Antibiotic resistance profiles}

All isolates were characterised for susceptibility to 11 antibiotics, including amoxicillin, chloramphenicol, ciprofloxacin, cefotaxime, gentamicin, kanamycin, nalidixic acid, streptomycin, sulphonamides, tetracycline and trimethoprim, using MIC Test strips (Liofilchem Diagnostics, Roseto degli Abruzzi, Italy). Interpretation was based on epidemiological cut-off values (ECOFFs) from the European Committee on Antimicrobial Susceptibility Testing (EUCAST) [12]. Salmonella ECOFFs for kanamycin and sulphonamides were listed as not determined, therefore the published E. coli ECOFF values were used for analysis. Antibiotic resistance of isolates identified as other Enterobacteriaceae was also interpreted using the reported E. coli ECOFFs. Once resistance to the 11 antibiotics was interpreted for all isolates, each unique resistance pattern was assigned a resistance profile number. These isolate data were used to determine the percentage of resistance to a given antibiotic, the frequency distribution of multidrug resistance and the geographic distribution of species-specific resistance profiles.

\section{Results and discussion}

This pilot antibiotic resistance surveillance survey encompassed 6 of the 12 large commercial Albanian poultry farms over a 3-month period from December 2013 to early February 2014. These farms process an estimated 800 000-1 200000 chickens per year. A total of 172 poultry samples were taken, yielding 91 bacterial isolates. Isolates were characterised by species (Salmonella, E. coli or other Enterobacteriaceae) and by susceptibility to 11 antibiotics. All Salmonella isolates obtained were identified as serotype Enteritidis by serotyping. There were 92 neck skin samples taken, which yielded 1 Salmonella, 9 E. coli and 22 Enterobacteriaceae isolates. Of the 20 stomach samples taken, 4 Salmonella, 7 E. coli and 4 Enterobacteriaceae isolates were obtained. Of the 6 intestine samples 
taken, no Salmonella, 1 E. coli and 3 Enterobacteriaceae isolates were obtained. Of the 12 stomach \& intestine samples taken, 2 Salmonella, 2 E. coli and 1 Enterobacteriaceae isolates were obtained. Of the 42 cloacal swabs taken, 4 Salmonella, 24 E. coli and 7 Enterobacteriaceae isolates were obtained.

\subsection{Bacterial isolates from Albanian poultry show high to extremely high levels of antibiotic resistance}

In 2012, the WHO Advisory Group on Integrated Surveillance of Antimicrobial Resistance (AGISAR) released the third revision of critically important and highly important antibiotics for human medicine [13]. High levels of resistance to these antibiotics in food animal bacterial isolates have serious implications for general public health, the safety of farm workers and their families, and the efficacy of veterinary treatments to control animal disease on-farm. Data in the current study (Table 1) show that there are reasons for concern about the antibiotic resistance situation in Albania.

This pilot study found extremely high levels of resistance ( $>70 \%$ as defined by the joint EFSA and ECDC EU summary report on antimicrobial resistance [14]) to 7 of the 11 antibiotics included (Table 1) among E. coli isolated from poultry. In contrast, in the results of the $2012 \mathrm{EU}$ summary report, no country reported $E$. coli from poultry exhibiting extremely high levels of resistance to any of the antibiotics tested [14]. The current study also found moderate levels of resistance to gentamicin and very high levels of resistance to streptomycin (Table 1), in contrast to low levels of resistance to gentamicin and high levels of resistance to streptomycin in the EU report [14]. The current study also found moderate levels of resistance to cefotaxime, which was similar to the level of resistance in $E$. coli isolates from poultry reported in the EU report [14]. Salmonella isolates from Albanian poultry showed extremely high levels of resistance to 5 of the 11 antibiotics included (Table 1). In contrast, no EU country reported Salmonella spp. isolates from poultry exhibiting extremely high levels of resistance to any of the antibiotics tested [14]. The EU summary did report low levels of resistance to cefotaxime and gentamicin [14], in contrast to the current study that found no Salmonella isolates exhibiting resistance to either of these antibiotics.

Currently, Albania has no systems in place for surveillance of veterinary antibiotic consumption, therefore it is not possible to confidently correlate usage patterns with the patterns of resistance found in this study. There are, however, some insights worth noting that were gained through private discussions with Albanian veterinary public health officials with practical knowledge and exposure to antibiotic usage on-farm. The first was regarding fluoroquinolone usage, which was said to be widespread and for prolonged periods on poultry farms, for therapeutic and preventive purposes, which correlates with the extremely high levels of resistance to nalidixic acid (81\%) and ciprofloxacin (85\%) in the isolates in this study. Conversely, gentamicin, kanamycin and cefotaxime are not known to be used in Albanian poultry, which may explain in part the low levels of resistance to the agents observed in this study. Lastly, it was indicated, whilst official numbers were lacking, that there was significant qualitative use, both individually and mixed, of antibiotics on Albanian farms. Further studies and data are clearly needed in the area of antibiotic consumption in Albania. 
Nevertheless, these observations suggest that antibiotic resistance levels reflect usage practices on poultry farms in Albania.

\subsection{Salmonella and Escherichia coli isolates exhibit resistance to multiple antibiotics}

Over $80 \%$ of the Salmonella isolates (9 of 11 isolates) showed resistance to at least four antibiotics studied and over $60 \%$ ( 7 of 11 isolates) showed resistance to at least seven of the antibiotics included; none were susceptible to all antibiotics tested (Fig. 2). In contrast, countries in the EU summary report did not report $>50 \%$ of the Salmonella poultry isolates displaying resistance to four or more antibiotics and not $>10 \%$ displaying resistance to seven or more [14]. The current study also found that $95 \%$ of the $E$. coli isolates (41 of 43 isolates) showed resistance to at least four antibiotics and over $80 \%$ ( 35 of 43 isolates) with resistance to at least seven antibiotics (Fig. 2). Again in contrast, no country in the EU summary reported $>40 \%$ of poultry E. coli isolates displaying resistance to four or more antibiotics, and none reported $>5 \%$ with resistance to seven or more antibiotics [14]. It is also interesting to note that high levels of multidrug resistance in other Enterobacteriaceae, suggesting that there is antibiotic resistance selection and maintenance across many bacterial species on Albanian poultry farms.

\subsection{Multidrug resistance profiles are geographically widespread in Albania poultry production}

A total of 42 unique resistance patterns were identified among the isolates included. Of these, 11 patterns were either found in multiple species or across multiple farms (Fig. 1). Profiles with multidrug resistance to the majority of antibiotics tested, such as profiles 5 and 17 that show resistance to 9 and 8 of the antibiotics, respectively, were found on at least one-half of the farms sampled. The distribution of these profiles across several species in a single location, e.g. profile 17 in Lushnjë or profile 20 in Elbasan (Fig. 1), could be indicative of on-farm horizontal transfer of antibiotic resistance genes between species. The presence of the same resistance profile in the same species across multiple locations, e.g. profile 5 and 17 in $E$. coli (Fig. 1), may also be indicative of clonal spread of antibiotic resistance across the country. Further genetic characterisation of these isolates would provide valuable information to determine the extent of horizontal gene transfer between species and whether these resistant isolates represent geographically disperse yet clonally related populations. These molecular capabilities were not available in the laboratory where the pilot programme's isolate characterisation was performed. Further work is needed to understand the spread of these multidrug-resistant strains among livestock farms and to prevent further spread and potential transmission to the public.

In summary, this pilot study indicates that antibiotic resistance among bacterial isolates from poultry is much higher in Albania compared with EU countries. Furthermore, many isolates also exhibited resistance to multiple antibiotics and are widely distributed across the poultry farms surveyed in Albania. These high levels of antibiotic resistance are significant and constitute a public health concern. Further research is needed to understand the levels of and linkages between on-farm 
antibiotic consumption and antibiotic resistance in Albania, as are policies that establish systems for the monitoring of these trends and that encourage prudent antibiotic use.

Acknowledgments: SDA would like to acknowledge the US Department of Agriculture Fellowship Program [2001-38420-20044], the Northeast Alliance for Graduate Education \& the Professoriate, and the National Institutes of Health (NIH) Initiative for Maximizing Student Development (IMSD) [grant GM099649] for their support.

Funding: This survey was conducted as part of a joint World Health Organization (WHO)-World Organization for Animal Health (OIE) project on 'National Human and Animal Health Systems Assessment Tools and Bridges', with financial support from the European Community, represented by the Commission of the European Communities under the European Commission Avian and Human Influenza Trust Fund, World Bank acting as administrator of the grant funds [Avian and Human Influenza Facility (AHIF)].

Competing interests: None declared.

Ethical approval: Not required. 


\section{References}

[1] Bell BG, Schellevis F, Stobberingh E, Goossens H, Pringle M. A systematic review and metaanalysis of the effects of antibiotic consumption on antibiotic resistance. BMC Infect Dis 2014;14:13.

[2] Levy SB, Marshall B. Antibacterial resistance worldwide: causes, challenges and responses. Nat Med 2004;10(12 Suppl):S122-9.

[3] van den Bogaard AE, Stobberingh EE. Epidemiology of resistance to antibiotics. Links between animals and humans. Int J Antimicrob Agents 2000;14:327-35.

[4] Alliance for the Prudent Use of Antibiotics; Avorn JL, Barrett JF, Davey PG, McEwen SA, O'Brien TF, Levy SB. Antibiotic resistance: synthesis of recommendations by expert policy groups. Boston, MA: World Health Organization; 2001. WHO/CDS/CSR/DRS/2001.10.

[5] Teuber M. Veterinary use and antibiotic resistance. Curr Opin Microbiol 2001;4:493-9.

[6] van de Sande-Bruinsma N, Grundmann H, Verloo D, Tiemersma E, Monen J, Goossens H, et al.; European Antimicrobial Resistance Surveillance System Group; European Surveillance of Antimicrobial Consumption Project Group. Antimicrobial drug use and resistance in Europe. Emerg Infect Dis 2008;14:1722-30.

[7] Landers TF, Cohen B, Wittum TE, Larson EL. A review of antibiotic use in food animals: perspective, policy, and potential. Public Health Rep 2012;127:4-22.

[8] Okeke IN, Laxminarayan R, Bhutta ZA, Duse AG, Jenkins P, O'Brien TF, et al. Antimicrobial resistance in developing countries. Part I: recent trends and current status. Lancet Infect Dis 2005;5:481-93.

[9] Jorgji K, Bebeci E, Apostoli P, Apostoli A. Evaluation of use of antibiotics without prescription among young adults in Albania case study: Tirana and Fier District. Hippokratia 2014;18:21720.

[10]Hoxha I, Malaj A, Malaj L. Antibiotic use in Albania between 2011 and 2012. J Infect Dev Ctries 2015;9:94-8.

[11]Bezhani E. The economic impact of agricultural product in the Albanian exports. Academic Journal of Interdisciplinary Studies 2013;2:6.

[12]European Committee on Antimicrobial Susceptibility Testing. MIC distributions and ECOFFs. http://www.eucast.org [accessed 28 October 2015].

[13]WHO Advisory Group on Integrated Surveillance of Antimicrobial Resistance (AGISAR). Critically important antimicrobials for human medicine. Geneva, Switzerland: World Health Organization; 2012.

[14]Eurosurveillance Editorial Team. European Union summary report on antimicrobial resistance in zoonotic and indicator bacteria from humans, animals and food 2012 published. Euro Surveill 2014;19:20748. 
Fig. 1. Distribution of antibiotic resistance profiles across Albanian poultry farms. Farm location followed by (number of bacterial isolates/total number of samples taken). For each farm, a box contains the profile number and the letters in parenthesis indicate isolate types with the profile: $\mathrm{e}$, Escherichia coli; s, Salmonella spp.; b, Enterobacteriaceae. Amx, amoxicillin; Chl, chloramphenicol; Cip, ciprofloxacin; Ctx, cefotaxime; Gen, gentamicin; Kan, kanamycin; Nal, nalidixic acid; Str, streptomycin; Sul, sulphonamides; Tet, tetracycline; Tmp, trimethoprim.

Fig. 2. Frequency distribution of bacterial isolates resistant to one to ten antibiotics from Albanian poultry. Numbers in parenthesis indicate total number of isolates. 


\section{Table 1}

Bacterial isolates showing antibiotic resistance

\begin{tabular}{|c|c|c|c|c|c|c|c|c|c|c|c|}
\hline \multirow[t]{2}{*}{ Species } & \multicolumn{11}{|c|}{ Percent resistance } \\
\hline & $\operatorname{AMX}^{a}$ & $\mathrm{CHL}^{\mathrm{b}}$ & CIP $^{a}$ & CTX $^{a}$ & GEN $^{a}$ & $\mathrm{KAN}^{\mathrm{a}}$ & $\mathrm{NAL}^{\mathrm{a}}$ & STR $^{a}$ & SUL $^{b}$ & TET $^{b}$ & TMP $^{b}$ \\
\hline Escherichia coli $(n=43)$ & 86 & 77 & 93 & 14 & 12 & 30 & 91 & 70 & 91 & 95 & 79 \\
\hline Enterobacteriaceae $(n=37)$ & 84 & 62 & 78 & 8 & 8 & 16 & 73 & 54 & 84 & 78 & 54 \\
\hline Salmonella spp. $(n=11)$ & 64 & 82 & 73 & 0 & 0 & 18 & 73 & 55 & 73 & 73 & 64 \\
\hline All isolates $(n=91)$ & 82 & 71 & 85 & 10 & 9 & 23 & 81 & 62 & 86 & 86 & 67 \\
\hline
\end{tabular}

AMX, amoxicillin; CHL, chloramphenicol; CIP, ciprofloxacin; CTX, cefotaxime; GEN, gentamicin; KAN, kanamycin; NAL, nalidixic acid; STR, streptomycin; SUL, sulphonamides; TET, tetracycline; TMP, trimethoprim.

${ }^{\text {a }}$ Critically important antibiotic as defined by the World Health Organization (WHO) [13].

${ }^{\mathrm{b}}$ Highly important antibiotic as defined by the WHO [13].

Terms used to describe the antimicrobial resistance levels are: rare, $<0.1 \%$; very low, $0.1-1 \%$; low, $>1-10 \%$; moderate, $>10-20 \%$; high, >20-50\%; very high, >50-70\%; and extremely high, >70\% [14]. 


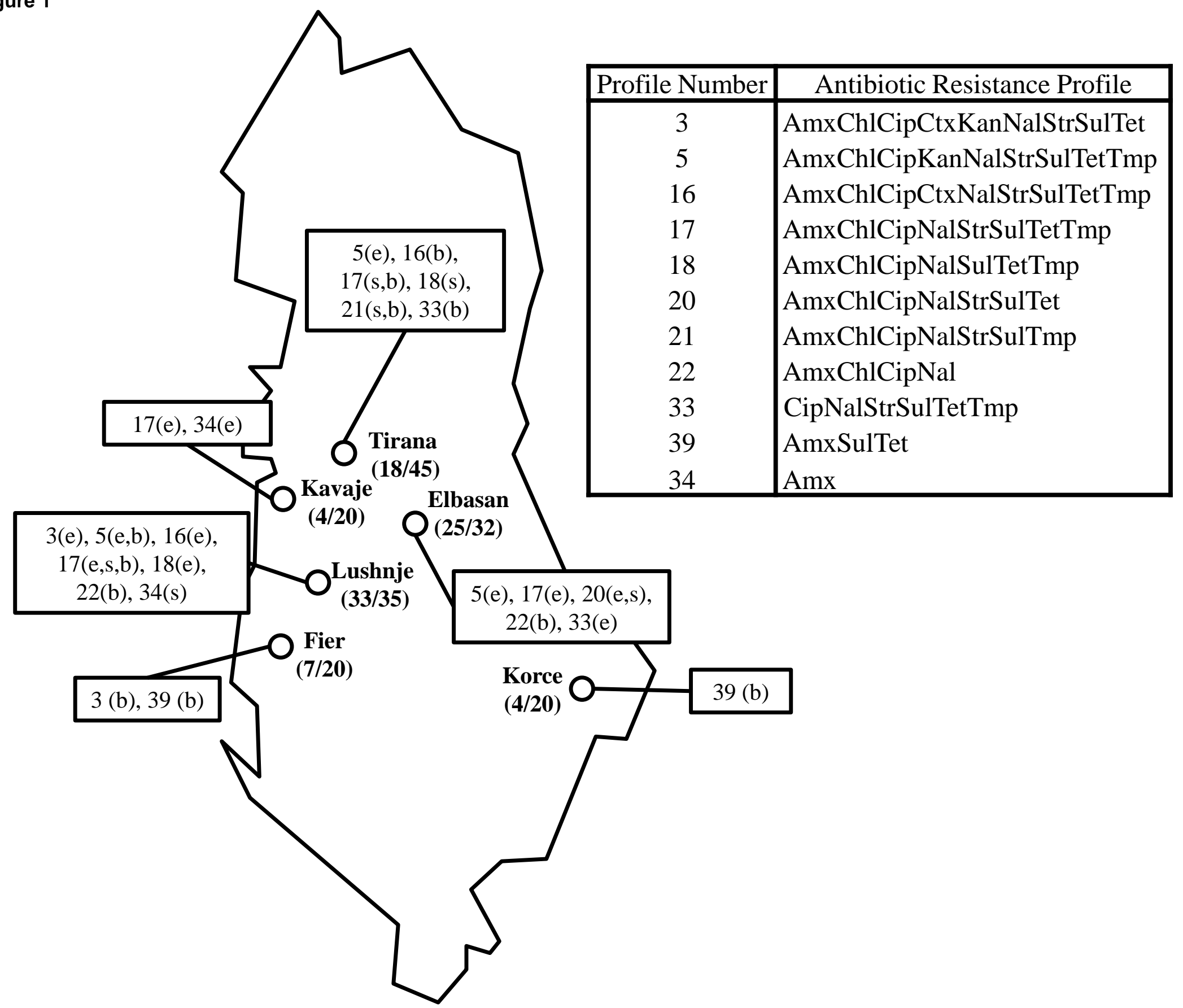


Salmonella spp. (11)

\section{Enterobacteriacae (37)}

E. coli (43)
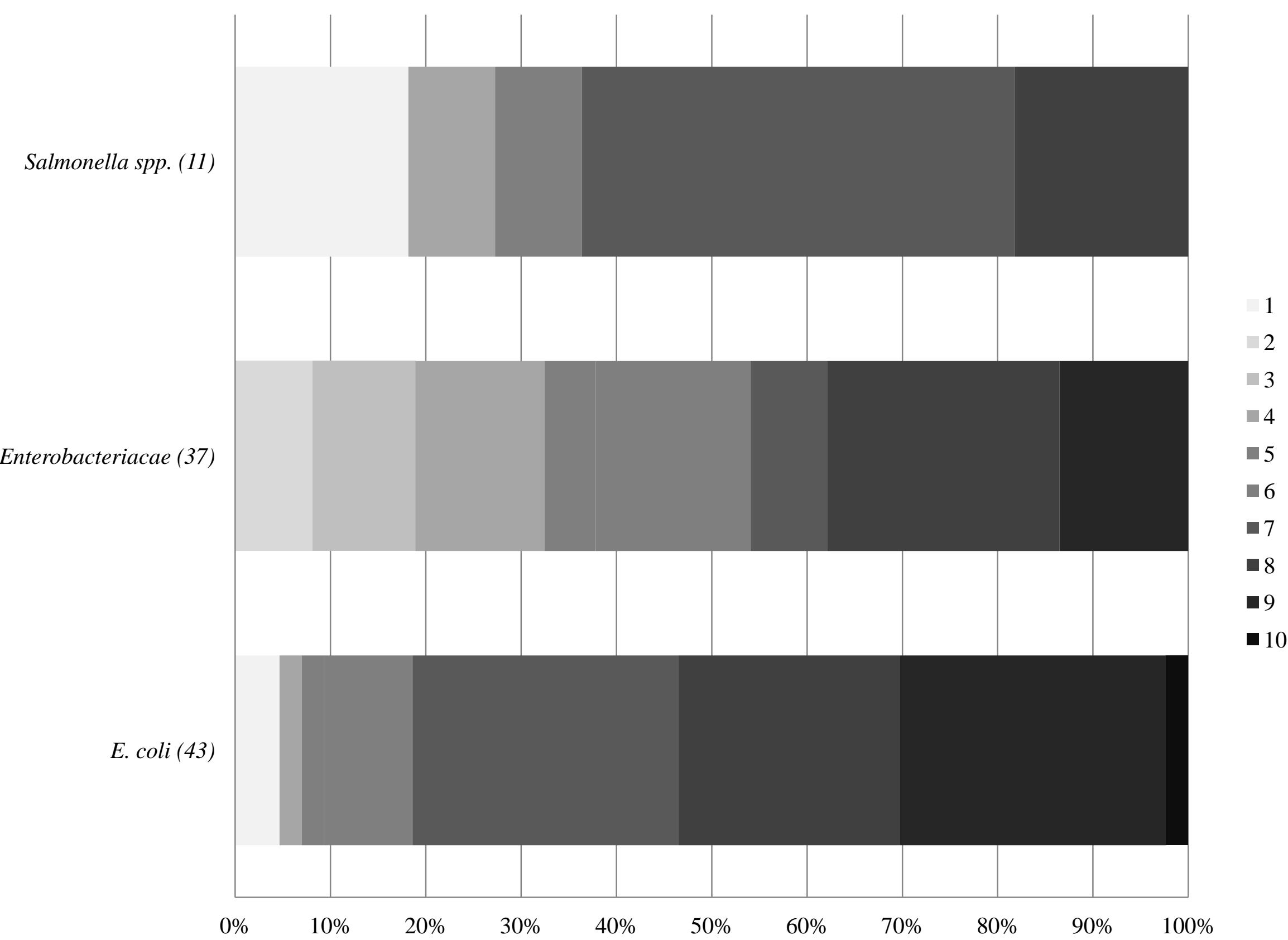